ESAIM: PROCEEDINGS, April 2007, Vol.17, 19-25

Alain Piétrus \& Michel H. Geoffroy, Editors

\title{
ASPLUND DECOMPOSITIONS OF MONOTONE OPERATORS
}

\author{
JONATHAN M. BORWEIN ${ }^{1}$
}

\begin{abstract}
This paper reviews what is known about the structure of monotone operators on nonreflexive spaces. It focusses on Asplund's interesting but neglected decomposition theorem.
\end{abstract}

\section{INTRODUCTION}

This paper is an extended version of my talk given at the associated 2004 conference in Guadeloupe. Further related matter can be found in [4]. In a largely forgotten 1968-1970 paper, Edgar Asplund, inter alia, provided a very provocative decomposition of a maximal monotone operator as the sum of a subgradient and an acyclic ("skew") part. In part, this forgetting is Asplund's fault. Titles of papers really do matter !

We start with Asplund's introduction:

In a life cut tragically short, some of Asplund's many other seminal contributions include:

(1) Generic existence results for nearest and farthest points to closed sets in Banach space.

(2) Central work on the still open Chebyshev problem of showing that a subset of Hilbert space admitting a unique nearest point for each point in the space must be convex.

(3) Asplund averaging of good (re)norms on a Banach space to produce a norm with two good properties.

(4) Generic differentiability of convex functions in 'SDS spaces'-now called Asplund spaces.

(5) Duality between smoothness and roundness (exposedness) properties for Banach spaces.

I intend to motivate revisiting Asplund's work on the 30th anniversary of his death, by asking how much we know about convex subgradients or monotone operators? For example, is a (bounded) linear mapping monotone iff its adjoint is? I shall then review monotonicity theory in non-reflexive spaces before presenting a modern version of an extension of one of Asplund's decomposition results.

I'll finish with some applications and extensions and pose some hard conjectures such as all monotone pathologies are realizable with 'skew' mappings.

\section{Preliminaries}

Definition 1.1. A mapping $T: X \rightarrow X^{*}$ is monotone if for all $x, y \in D:=\operatorname{dom} T$

$$
\left\langle y^{*}-x^{*}, y-x\right\rangle \geq 0
$$

for all $x^{*} \in T(x), y^{*} \in T(y)$. We say $T$ is skew if $T$ and $-T$ are both monotone.

\footnotetext{
1 Canada Research Chair in IT, Dalhousie University, Halifax, Nova Scotia, Canada.
} 
Definition 1.2. A (multi-)function $T: D \subset X \rightarrow X^{*}$ is $n$-monotone if

$$
\left\langle T\left(x_{1}\right), x_{1}-x_{n}\right\rangle+\sum_{k=2}^{n}\left\langle T\left(x_{k}\right), x_{k}-x_{k-1}\right\rangle \geq 0
$$

holds for all $\left(x_{1}, \ldots, x_{n}\right) \in D^{n}$, and $T$ is cyclically monotone if it is $n$-monotone for all $n$.

These $n$-monotone classes are distinct as shown by Asplund in [1]. Our interest in cyclic monotonicity is largely motivated by the following:

Theorem 1.3. (Rockafellar, [9]) Given a relation $\rho$ on $X \times X^{*}$, there exists a closed convex function $f$ on $X$ such that $\partial f \supset \rho$ if and only if $\rho$ is cyclically monotone.

Throughout, $X$ is a Banach space and $\partial f$ is the convex subdifferential familiar from convex analysis:

$$
\partial f(x)=\left\{x^{*} \in X^{*}:\left\langle x^{*}, y-x\right\rangle+f(x) \leq f(y) \forall y \in X\right\} .
$$

Thus, $\partial f$ is maximally cyclically monotone iff it is a subgradient of a closed convex function. Skew and monotone easily implies acyclic and the converse holds when $T$ is linear.

\section{Convex Subgradients}

One must take care, even in separable Hilbert space and even with convex closed functions.

Example 2.1. ( [5]) A proper lower semicontinuous convex function, $f$, on separable Hilbert space with the graph of $\partial f$ not nor $\mathrm{m} \times$ bounded-weakstar closed.

Let $E:=\ell_{2}(\mathbb{N})$ and define

$$
e_{p, m}:=\frac{1}{p}\left(e_{p}+e_{p^{m}}\right), \quad e_{p, m}^{*}:=e_{p}^{*}+(p-1) e_{p^{m}}^{*}
$$

for $m, p, r, s \in \mathbb{N}, m \geq 2$ and $p$ prime. We have $\left\langle e_{p, m}^{*}, e_{p^{\prime}, m^{\prime}}\right\rangle=0$ if $p \neq p^{\prime},=1 / p$ if $p=p^{\prime}, m \neq m^{\prime}$ and $=1$ if $p=p^{\prime}, m=m^{\prime}$. For $x \in E$ define

$$
f(x):=\max _{m>1, p}\left(\left\langle e_{1}^{*}, x\right\rangle+1, \sup \left\{\left\langle e_{p, m}^{*}, x\right\rangle\right\}\right)
$$

so $f$ is a proper lsc convex function on $E$.

To see that $f$ is as claimed we argue as follows:

(1) Then $f(0)=f\left(e_{p, m}\right)=1, f\left(-e_{1}\right)=0$ and $f(x) \geq\left\langle e_{p, m}^{*}, x\right\rangle$ for $x \in E$, for $m \geq 2$ and $p$ prime. So, $e_{p, m}^{*} \in \partial f\left(e_{p, m}\right)$.

(2) Also $0^{*} \notin \partial f(0)$, since $0^{*} \in \partial f(0)$ is equivalent to $f(x)-f(0) \geq 0$ for all $x \in E$, which fails for $x=-e_{1}$. Thus $\left(0,0^{*}\right)$ is not in the graph of $\partial f$.

(3) The graph of $\partial f$ is not norm $\times$ bw closed: $\left(0,0^{*}\right)$ is in the norm $\times$ bw closure of

$$
\left\{\left(e_{p, m}, e_{p, m}^{*}\right): \quad m \geq 2, p \text { prime }\right\} \subseteq \operatorname{graph} \partial f .
$$

Informally, this is true since $e_{p, m}$ tends in norm to 0 for large $p$, and also $0^{*}$ is a bw-cluster point of the $e_{p, m}^{*}$.

Example 2.2. ( [5]) Less instructively, but more easily, one may use $E=\ell^{2}([0,1]$ ) (which is non-separable) and

$$
f_{1}(x):=\max _{0<r \leq 1}\left(\left\langle e_{0}, x\right\rangle+1, \sup \left\{r^{-1}\left\langle e_{r}, x\right\rangle\right\}\right) .
$$


Before we used an unbounded sequence with a $\mathrm{w}^{*}$-cluster point; here that $\left\{r^{-1} e_{r}: 0<r \leq 1\right\}$ has $0^{*}$ in its bw* closure This example was built after Isac Namioka noted the bw* topology is nastier than Fitzpatrick and I knew. The idea of the proof originates with Von Neumann.

More generally, we have:

Theorem 2.3. ( [5]) Let $E$ be a Banach space. The following are equivalent:

i) $E$ is finite dimensional.

ii) The graph of $\partial f$ is norm $\times b w^{*}$ closed for each closed convex $f$ on $E$.

iii) The graph of each maximal monotone $T$ on $E$ is norm $\times b w^{*}$ closed.

Thus, all limiting constructions of generalized gradients, that capture the convex subdifferential, must fail to be closed for general lower semi-continuous mappings, unless they are locally bounded.

Question. Is Theorem 2.3 true if int $D(T)$ (int dom $f$ ) is required to be non-empty? Fitzpatrick and I conjectured that it is not. That is, we think it possible, at least in reflexive space, that:

The graph of every maximal monotone $T$ with $D(T)$ having nonempty interior interior is norm $\times$ bw* closed.

We tried quite hard but failed to build a counter-example.

\section{The 'Zoo' of Monotone Operators} dual.

Suppose $T$ is a (monotone) set-valued map from $X$ to $X^{*}$. We may consider various extensions to the second

Definition 3.1. Define set-valued maps $T_{1}, T_{0}, \bar{T}$ from $X^{* *}$ to $X^{*}$ via:

(1) $\left(x^{* *}, x^{*}\right) \in \operatorname{Gr}\left(T_{1}\right)$, if there is a bounded net $\left(x_{\alpha}, x_{\alpha}^{*}\right)$ in $\operatorname{Gr}(T)$ with

$x_{\alpha} \rightarrow_{*} x^{* *}$ and $x_{\alpha}^{*} \rightarrow x^{*}$.

(2) $\left(x^{* *}, x^{*}\right) \in \operatorname{Gr}\left(T_{0}\right)$, if

$$
\inf _{\left(y, y^{*}\right) \in \operatorname{Gr}(T)}\left\langle y^{*}-x^{*}, y-x^{* *}\right\rangle=0 \text {. }
$$

(3) $\left(x^{* *}, x^{*}\right) \in \operatorname{Gr}(\bar{T})$, if

$$
\inf _{\left(y, y^{*}\right) \in \operatorname{Gr}(T)}\left\langle y^{*}-x^{*}, y-x^{* *}\right\rangle \geq 0 \text {. }
$$

We are now able to we make the following definitions:

Definition 3.2. $\quad$ i) $T$ is dense type (D) if $T_{1}=\bar{T}$, [8].

ii) $T$ is range-dense type (WD) if for every $x^{*} \in \mathrm{R}(\bar{T})$, there is a bounded net $\left(x_{\alpha}, x_{\alpha}^{*}\right) \in \operatorname{Gr}(T)$ with $x_{\alpha}^{*} \rightarrow x^{*},[10]$.

iii) $T$ is type negative infimum $(N I)$ if $\inf _{\left(y, y^{*}\right) \in \operatorname{Gr}(T)}\left\langle y^{*}-x^{*}, y-x^{* *}\right\rangle \leq 0$, for all $\left(x^{* *}, x^{*}\right) \in X^{* *} \times X^{*},[10]$.

iv) $T$ is locally maximal monotone $(F P)$, if $\left(\mathrm{Gr}^{-1}\right) \cap(V \times X)$ is maximal monotone in $V \times X$, for each convex open $V$ in $X^{*}$ with $V \cap \mathrm{R}(T) \neq \emptyset[7]$.

v) $T$ is unique, if all maximal monotone extensions of $T$ in $X^{* *} \times X^{*}$ coincide.

$\ldots$ and there are other many classes (see $[2,4,10]$.

\subsection{Life in Reflexive Spaces}

In every Banach space, convex subgradients have all these properties. Moreover, maximal monotone and dense type, or locally maximal monotone implies maximal monotone. The converses of these and like statements hold in reflexive space, and are often but not always easily established. Linear examples show this may fail in some non-reflexive spaces (as described below).

In reflexive space the theory is really quite good - at least when some core condition is in force. Sum and composition rules and domain/range behavior in the non-reflexive case is complicated and subtle. Generally, things are only partially understood with very few counter-examples. In part, this is because we can, unfortunately, say a lot about the linear case: 


\section{The Truth About Linear Maps}

Proposition 4.1. ( [2]) Suppose $T$ is a continuous linear operator from $X$ to $X^{*}$. Then $T$ is weakly compact if and only if $T_{1}=T^{* *}$. Moreover, the following are equivalent: (i) $T$ is positive; (ii) $T$ is monotone; (iii) $T$ is maximal monotone.

We rely also on the following easy-to-prove yet immensely useful decomposition principle.

Proposition 4.2. ( [2]) Suppose $T$ is a continuous linear operator from $X$ to $X^{*}$. Then $T$ can be written uniquely as the sum of two continuous linear operators, $T=P+S$, where $P$ is symmetric and $S$ is skew:

$$
P x=\frac{1}{2} T x+\frac{1}{2} T^{*} x, \quad S x=\frac{1}{2} T x-\frac{1}{2} T^{*} x, \quad \forall x \in X .
$$

Note that, $P$ (resp. S) is the symmetric part (resp. skew part) of $T$.

We are now ready for the main linear result.

Theorem 4.3. ([2]) Suppose $T$ is a continuous linear operator from $X$ to $X^{*}$. Then the following are equivalent:

i) $T$ is monotone and of dense type, or range-dense type, or type (NI).

ii) $T$ is locally maximal monotone.

iii) $T^{*}$ is monotone.

(iv) $P$ and $S^{*}$ are monotone.

(v) $P$ is monotone and $S$ is of dense type, or range-dense type, or type (NI), or locally maximal monotone.

Actually, closed and densely defined suffices as shown by Phelps and Simons, [10]. This slightly mind-numbing result says "Linear maps can not distinguish any of the classes." In particular, there is a pathological positive map if and only if it is not the case that $X$ is such that every bounded map from $X$ to $X^{*}$ is weakly compact ( $X$ is a so-called cms space) [2].

Moreover, if $X$ is a Banach lattice, then the adjoint of every positive (resp. skew) map is positive (skew) iff $\mathrm{X}$ contains no isometric copy of $\ell_{1}$, as is the case for $C[0,1]$.

The - only - two fundamental examples are due to Gossez and to Fitzpatrick \& Phelps. We next describe general geometric way to analyze linear monotone operators that was given in [2, Theorem 5.1].

\subsection{Good and So-So Operators}

There are three mutually exclusive cases: $T$ is "good": $S^{*}$ and $-S^{*}$ are monotone; $T$ is "so-so": one of $S^{*}$ or $-S^{*}$ is monotone; $T$ is "bad": neither $S^{*}$ nor $-S^{*}$ is monotone.

Here is an example of a "so-so" operator.

Example 4.4. (Gossez) Consider $G: \ell_{1} \rightarrow \ell_{\infty}$ with

$$
(G x)_{n}:=-\sum_{k<n} x_{k}+\sum_{k>n} x_{k}, \quad \forall x=\left(x_{k}\right) \in \ell_{1}, n \in \mathbb{N}
$$

Then $G$ and $-G$ are skew operators from $\ell_{1}$ to $\ell_{\infty}$ and $G^{*}$ is not monotone but $-G^{*}$ is and so both of dense type and locally maximal monotone.

\subsection{A Bad Operator}

Somewhat surprisingly, the "continuous" version of the (negative) Gossez operator is "bad".

Example 4.5. (Fitzpatrick and Phelps) Define $F: L_{1}[0,1] \rightarrow L_{\infty}[0,1]$ by

$$
(F x)(t):=\int_{0}^{t} x(s) d s-\int_{t}^{1} x(s) d s, \forall x \in L_{1}[0,1],
$$


for $t \in[0,1]$.

Then $F,-F$ are skew from $L_{1}[0,1]$ to $L_{\infty}[0,1]$ but neither $F^{*}$ nor $-F^{*}$ is monotone. Consequently, neither $F$ or $-F$ is of type (NI) nor locally maximal monotone.

\section{A Cyclic-Acyclic Decomposition}

Adding a regularizing term (duality map, subgradient) provably can not worsen things, [2]. We have effectively exhausted all known counter-examples: a general conjecture is brewing. Studying a reworked version of Asplund's decomposition result in [1] to the stew reinforces this sense.

Definition 5.1. A maximal monotone operator $A$ is acyclic if whenever $A=\partial g+M$ with $M$ maximal monotone and $g$ closed and convex then $g$ is necessarily a linear function.

Theorem 5.2. (Asplund, [1, Theorem 2].) Suppose that $T$ is a single-valued maximal monotone operator defined on a set $D$ in $X^{*}$ (resp. X) whose weak* (resp. weak) closure has norm interior, and is norm-to-norm continuous (on D) at a point in this interior. Then, there is a convex subgradient operator $G=\partial f$ and an acyclically monotone mapping A such that

$$
T=\partial f+A .
$$

We note that linear mappings are acyclic iff they are skew. In finite dimensions, one may directly characterize when a $C^{1}$ monotone operator is the sum of a convex gradient and a skew linear mapping, [6].

The basic reason why we may decompose $T$ as $T=\partial f+A$ is: a delicate 'Zornification' to obtain a maximally cyclic part. The interiority condition enforces convergence of cyclically increasing nets. Rockafellar's result of Theorem 1.3 makes this cyclic part a convex subgradient while maximality (in the cyclic order) forces the remainder to be acyclically monotone.

We next elaborate Asplund's approach in [1] to provide a very general decomposition result. We begin by observing that every 3 -monotone operator such that $0 \in T(0)$ has the property that

$$
\left\langle x, x^{*}\right\rangle+\left\langle y, y^{*}\right\rangle \geq\left\langle x, y^{*}\right\rangle
$$

whenever $x^{*} \in T(x)$ and $y^{*} \in T(y)$. We will call a monotone operator satisfying (1), $3^{-}$-monotone, and write $T \geq_{N} S$ when $T=S+R$ with $R$ being $N$-monotone. Likewise we write $T \geq_{\omega_{0}} S$ when $R$ is cyclically monotone.

Proposition 5.3. Let $N$ be one of $3^{-}, 3,4, \ldots$ or $\omega_{0}$. Consider an increasing net of monotone operators on a Banach space $X$, satisfying

$$
0 \leq_{N} T_{\alpha} \leq_{N} T_{\beta} \leq_{2} T
$$

whenever $\alpha<\beta \in \mathcal{A}$.

Suppose that $0 \in T_{\alpha}(0), 0 \in T(0)$ and that $0 \in$ coredomT. Then there is a $N$-monotone operator $T_{\mathcal{A}}$ such that $T_{\alpha} \leq_{N} T_{\mathcal{A}} \leq_{2} T$, for all $\alpha \in \mathcal{A}$.

Proof. We first give the details in the single-valued case. Since $0 \leq_{2} T_{\alpha} \leq_{2} T_{\beta} \leq_{2} T$, while $T(0)=0=T_{\alpha}(0)$, we have

$$
0 \leq\left\langle x, T_{\alpha}(x)\right\rangle \leq\left\langle x, T_{\beta}(x)\right\rangle \leq\langle x, T(x)\rangle,
$$

for all $x$ in domT. This shows that $\left\langle x, T_{\alpha}(x)\right\rangle$ converges as $\alpha$ goes to $\infty$.

Fix $\varepsilon>0$ and $M>0$ with $T\left(\varepsilon B_{X}\right) \subset M B_{X^{*}}$. We write $T_{\beta \alpha}=T_{\beta}-T_{\alpha}$ for $\beta>\alpha$, so that $\left\langle T_{\beta \alpha} x, x\right\rangle \rightarrow 0$ for $x \in \operatorname{domT}$ as $\alpha, \beta$ go to $\infty$.

We appeal to (1) to obtain

$$
\left\langle x, T_{\beta \alpha}(x)\right\rangle+\left\langle y, T_{\beta \alpha}(y)\right\rangle \geq\left\langle T_{\beta \alpha}(x), y\right\rangle
$$

for $x, y \in \operatorname{domT}$. Also, $0 \leq\left\langle x, T_{\beta \alpha}(x)\right\rangle \leq \varepsilon$ for $\beta>\alpha>\gamma(x)$ for all $x \in \operatorname{domT}$. 
Now, $0 \leq\left\langle y, T_{\beta \alpha}(y)\right\rangle \leq\langle y, T(y)\rangle \leq \varepsilon M$ for $\|y\| \leq \varepsilon$. Thus, for $\|y\| \leq \varepsilon$ and $\beta>\alpha>\gamma(x)$ we have

$$
\begin{aligned}
\varepsilon(M+1) & \geq\left\langle x, T_{\beta \alpha}(x)\right\rangle+\langle y, T(y)\rangle \\
& \geq\left\langle x, T_{\beta \alpha}(x)\right\rangle+\left\langle y, T_{\beta \alpha}(y)\right\rangle \\
& \geq\left\langle y, T_{\beta \alpha}(x)\right\rangle,
\end{aligned}
$$

from which we obtain $\left\|T_{\beta \alpha}(x)\right\| \leq M+1$ for all $x \in \operatorname{domT}$, while $\left\langle y, T_{\beta \alpha}(x)\right\rangle \rightarrow 0$ for all $y \in X$. We conclude that $\left\{T_{\alpha}(x)\right\}_{\alpha \in \mathcal{A}}$ is a norm-bounded weak-star Cauchy net and so weak-star convergent to the desired $N$-monotone limit $T_{\mathcal{A}}(x)$.

In the general case we may still use (1) to deduce that $T_{\beta}=T_{\alpha}+T_{\beta \alpha}$ where (i) $T_{\beta \alpha} \subset(M+1) B_{X^{*}}$ and (ii) for each $t_{\beta \alpha}^{*} \in T_{\beta \alpha}$ one has $t_{\beta \alpha}^{*} \neg^{*} 0$ as $\alpha$ and $\beta \rightarrow \infty$. The conclusion follows as before, but is somewhat more technical.

We comment that $0 \leq_{2}(n y, n x) \leq_{2}(y, x)$ for $n \in \mathbb{N}$, shows the need for $(1)$ in the deduction that $T_{\beta \alpha}(x)$ are equi-norm bounded.

We can now provide the promised extension of Asplund's original idea:

Theorem 5.4. Suppose that $T$ is a maximal monotone operator on a Banach space with $\operatorname{dom} T$ having nonempty interior. Then $T$ may be decomposed as $T=\partial f+A$, where $f$ is closed and convex while $A$ is acyclic.

Proof. We normalize so $0 \in T(0)$ and apply Zorn's lemma to the set of cyclically monotone operators $\mathcal{C}:=$ $\left\{C: 0 \leq_{\omega_{0}} C \leq_{2} T, 0 \in C(0)\right\}$ in the cyclic order. By Proposition 5.3 every chain in $\mathcal{C}$ has a cyclically monotone upper-bound. Consider such a maximal $\bar{C}$ with $0 \leq_{\omega_{0}} \bar{C} \leq_{2} T$. Hence $T=\bar{C}+A$ where by construction $A$ is acyclic. Now, $T=\bar{C}+A \subset \partial f+A$, by Rockafellar's result (Theorem 1.3). Since $T$ is maximal the decomposition is as asserted.

This and related results in $[1,4]$ are entirely existential: how can one prove Theorem 5.4 constructively in finite dimensions? What is the decomposition for such simple monotone maps such as $(x, y) \mapsto(\sinh (x)-$ $\left.\alpha y^{2} / 2, \sinh (x)-\alpha x^{2} / 2\right)$ which is monotone exactly for $\alpha \geq-2 / \sqrt{x_{0}^{2}-1} \sim 0.7544 \ldots$ with $x_{0}$ the smallest fixed point of coth?

\section{A Class of Indecomposable Monotone Functions}

Partially because the acyclic part is somewhat illusive, let us say that $T$ is decomposable if $T=\partial f+S$ where $S$ is skew and linear, or equivalently if the acyclic part in Theorem 5.4 is actually skew. If this is not the case we say $T$ is indecomposable.

We associate a function

$$
f_{T}(x):=\int_{0}^{1}\langle x, T(t x)\rangle d t
$$

with a (single-valued) monotone $C^{1}$ operator with 0 in the core of its domain. Then $T$ is decomposable if and only if $T-\nabla f_{T}$ is skew. In this case $f_{T}$ is convex, see [6].

For simplicity we state the next result in $\mathbf{R}^{\mathbf{2}}$.

Claim 6.1. ( [6]) Let $g \geq 0$ be a non-constant integrable real function such that with $g(x) \geq 1=g(0)$ or $g(x) \leq 1=g(0)$. Let $G(x):=\int_{0}^{x} g$ and $K(x):=\int_{0}^{x}\{(1+g) / 2\}^{2}$. Then

(1) $T(x, y):=(K(x)-G(y), K(y)-G(x))$ is $C^{1}$ and monotone.

(2) $T$ is indecomposable.

Proof. (Outline) Monotonicity follows from checking that the gradient is semi-definite, and indecomposability from checking that the mixed-partials are not the same.

We note that for $x=0$ or $y=0$, $\operatorname{det} \nabla T(x, y)=0$ which is prerequisite for $T$ to be acyclic. Is it? 
Example 6.2. If $g:=x^{2}+1$ then

$$
T(x, y)=\left(x+1 / 20 x^{5}+1 / 3 x^{3}-1 / 3 y^{3}-y, y+1 / 20 y^{5}+1 / 3 y^{3}-1 / 3 x^{3}-x\right)
$$

and

$$
f_{T}(x, y)=\frac{1}{120} x^{6}+\frac{1}{120} y^{6}+\frac{1}{12} x^{4}+\frac{1}{12} y^{4}-\frac{1}{12} x y^{3}-\frac{1}{12} y x^{3}+\frac{1}{2} x^{2}-x y+\frac{1}{2} y^{2} .
$$

We note that $f_{T}$ is not convex, [6].

\section{My Subgradient Conjectures}

So after many years and failures I have the following

\section{Structure Conjectures.}

- All known "nice" monotone classes, $\mathcal{M}$, are closed under addition of a subgradient:

$$
\mathcal{M}+\partial f \subset \mathcal{M}
$$

- All these "nice" classes, $\mathcal{M}$, coincide.

- "Bad" operators can seemingly always be realized by skew (linear) operators.

In short, subgradients and 'skews' are apparently ubiquitous. In what precise sense are they the extreme points of the class of maximal monotone operators?

\section{REFERENCES}

[1] E. Asplund, A monotone convergence theorem for sequences of nonlinear mappings, Proceedings of Symposia in Pure Mathematics. Vol. 18, Part 1, (1970), 1-9.

[2] H. H. Bauschke and J. M. Borwein, Continuous Linear Monotone Operators on Banach Spaces, Pacific J. Math. 189 (1999), no. $1,1-20$.

[3] Heinz H. Bauschke, Jonathan M. Borwein and Patrick L. Combettes, Essential smoothness, essential strict convexity, and convex functions of Legendre type in Banach spaces, Communications in Contemporary Mathematics, 3 (2001), 615-648. [CECM Preprint 00:156].

[4] J. M. Borwein, Monotone Operators via Convex Analysis, J. Convex Analysis, in press. [Dalhousie D-drive Preprint 281].

[5] J.M. Borwein, S.P. FitzPatrick and R. Girgensohn, Subdifferentials whose graphs are not bounded $\times$ weak-star closed, Can. Math. Bull., 46 (2003), 538-545 [SFU CoLab Preprint 01:175].

[6] J. M. Borwein and H. Wiersma, Decomposable Monotone Operators, in preparation.

[7] S. FitzPatrick And R.R. Phelps R, Some properties of Maximal monotone operators on nonreflexive Banach spaces, SetValued Anal., 3 (1995), 51-69.

[8] J.-P. Gossez, Opérateurs monotones non linéaires dans les espaces de Banach non réflexifs, J. Math. Anal. Appl., 34 (1971), 371-395.

[9] R. T. Rockafellar, Characterization of the subdifferentials of convex functions, Pacific J. Math. 17 (1966), 497-510.

[10] S. Simons, Minimax and Monotonicity, Lecture Notes in Math. 1693, Springer-Verlag, New York, 1998. 